\title{
Potential Role of Wearable, Ambulatory and Home Monitoring Systems for Patients with Neurodegenerative Diseases and Their Caregivers
}

\author{
Paolo Meriggi, Paolo Castiglioni, Francesco Rizzo, Valerio Gower, Renzo Andrich, \\ Marco Rabuffetti, Maurizio Ferrarin, Marco Di Rienzo \\ Polo Tecnologico (Biomedical Technology Department) \\ Fondazione Don Carlo Gnocchi Onlus, \\ Milano, Italy \\ email: pmeriggi@dongnocchi.it
}

\begin{abstract}
Neurodegenerative diseases (NDD) require a constant care and attention in pharmacological therapy and rehabilitation, exercises administration, functional assessments and management of daily life. In this paper, we discuss a possible role of pervasive solutions with respect to NDD, on the basis of the overall progress of ICT and on the experience achieved in our department in three fields: Assistive Technology, NDD quantitative assessment, and design and development of wearable devices.
\end{abstract}

Keywords: neurodegenerative diseases, NDD, wearable systems, pervasive computing, ambulatory monitoring, multimodal interfaces

\section{INTRODUCTION}

Neurodegenerative diseases (NDD) are characterized by progressive decline and loss of cognitive and/or motor functions, which may occur over long-term period. Care of NDD patients frequently requires quite extensive efforts by their caregivers, often spouses or other family members, leading them to poor quality of life [1].

After the onset of the disease (diagnosis and subsequent definition of the therapeutic plan), patient's follow-up is usually based on periodic health assessments performed in hospitals or clinical labs. Apart from these periodic visits, most of the contacts with clinicians are related to adaptations of the pharmacological treatment due to worsening or major changes in the patient's conditions.

Recent studies emphasized that this approach still leaves a number of unmet needs in caregivers [2], and difficulties in coping with NDDs for patients [3]. In fact, the usual approach is mainly based on pharmacological treatments (often palliative), sometimes on rehabilitation physical therapies, and, seldom, on psychological support. Thus, after the initial intervention, the follow-up assessments may be too sparse to allow an early detection of subclinical worsening in patients' functions, or to foresee degradations in patients and caregivers' quality of life and social inclusion.

NDD patients are often supported by a wide range of solutions, named Assistive Technologies (AT), spreading form from walking canes to eyetracking systems. Most of these solutions are targeted to very specific needs of the patients and they are not usually meant to be networked. According to one of the most used definition of Assistive Technology (AT), these solutions consists in '... any item, piece of equipment, or product system whether acquired commercially off the shelf, modified, or customized, that is used to increase, maintain, or improve functional capabilities of individuals with disabilities' [4].

Despite the growing interest around AT solutions, there seems to be quite a gap between NDD patient's side (i.e. patients and caregivers) and those in charge of patients' health (clinicians, therapists, etc.). Still this gap does not seem to be sufficiently addressed by the growing number of portable, distributed and pervasive solutions available

In this paper, we report about the experience achieved in our department in different areas. From this experience, we propose an ICT architecture that might be helpful in the daily coping with NDDs, by exploiting the use of pervasive technologies. In order to enhance the robustness of such an approach, it is important also to exploit the experience gathered within the field of assistive technologies, by implementing suitable assessment efforts on usability, efficacy and efficiency, from the design stage to the deployment.

\section{THE EXPERIENCE AT POLO TeCNOLOGICO,} FONDAZIONE DON CARLO GNOCCHI

Fondazione Don Carlo Gnocchi is a no-profit foundation operating since 1951 [5], and one of the biggest private institution in Italy in rehabilitation, elderly and disabled care. Neurodegenerative diseases are among the target pathologies for the core activities of the foundation [6]. Within the Fondazione Don Gnocchi, Polo Tecnologico (Biomedical Technology Department), formerly Centro di Bioingegneria, has been actively involved in these activities on different levels. One level regards the Assistive Technologies Area and consists in the study and assessment of AT, in terms of ergonomics, usability, and cost-effectiveness [7]. Another level regards the Neuromotor Area and consists in the evaluation and development of technological solutions, and of the related clinical protocols, for assessment and rehabilitation of NDD's patients. Beside these activities, others more generally dealing with the rehabilitation (in 
particular the cardiovascular one) are focused on design and development of wearable devices and home monitoring architectures (Wearable Technologies Area). In the next paragraphs, we briefly report about these three areas, showing how these experiences may converge in a novel pervasive architecture with the aim of supporting NDDs patients and caregivers.

\section{A. Assistive Technology studies}

In the past decades, a number of studies have been performed within the Assistive Technologies Area, according to three research lines: technologies for information and education, technology assessment and ambient assisted living.

The first line (technology for the information and education) refers to the design and development of methods to support the distribution of information and distance education for the several actors involved in the research and deployment of assistive technologies (disabled persons, caregivers, operators, industry and institutional stakeholders, researchers). Within this research line, the Italian information portal on assistive technologies has been developed (www.portale.siva.it). Moreover, the AT area is currently leading the European Information Network on Assistive Technology (www.eastin.info), also promoting the Association for the Advancement of Assistive Technologies in Europe (AAATE - www.aaate.net) as National Contact.

The second research line (technology assessment) is focused on design and validation of methods for the evaluation of technical quality (safety, robustness, reliability, usability), efficacy (goal achievements), usefulness (user perceived value) and efficiency (investments/results ratio) of the AT solutions

The last research line (ambient assisted living) is related to design and evaluation of devices, systems and methodologies aimed at improving the independence of people with disabilities in their living environment. In 2007, a Smart Home has been realized, within the Occupational Therapy service of Fondazione Don Carlo Gnocchi. The Smart Home is also a testbed for the evaluation of innovative AT solutions in a real home setting [8].

\section{B. NDD Neuromotor Studies}

Several researches related to quantitative assessments of NDDs have been carried out at the Biomedical Technology Department. They have been focused on Parkinson Disease (PD) [9] [10] [11], Cerebellar Ataxia (CA) [12], Multiple Sclerosis [13], Alzheimer Disease [14], Charcot-MarieTooth disease (CMT) [15], and Amyotrophic Lateral Sclerosis (ALS). Most of these studies have been performed by means of complex lab devices (such as Optoelectronic Motion Capture Systems - Smart, BTS, Italy -, Force Platform Systems - Kistler, Switzerland -, and robotic arms), but also by means of compact appliances (such as portable EMG systems, tablet and touch-screen computers [16], and eye-trackers). In Fig. 1 we may see an example of acquisition performed by the optoelectronic system.

Results of these researches underline the potentials of advanced technological devices in realizing interesting diagnostic, clinical and therapeutical protocols. These devices and systems seem to be highly sensitive tools in the diagnostic process, and very effective in the therapeutic and rehabilitation treatments. Moreover, such lab systems may also be proficiently used as reference for portable solutions meant to be used in the daily routine of patients.
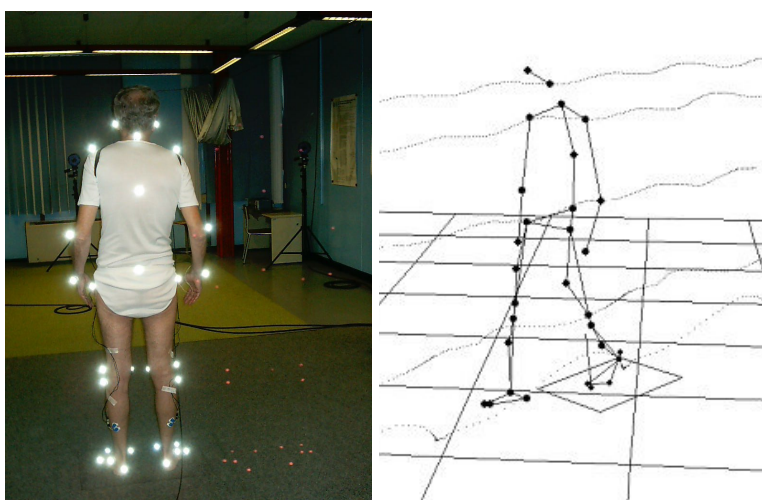

Figure 1. Example of quantitative analysis performed with the optoelectronic system: a subject wearing markers (left panel) and the results of the acquired trajectories (right panel) while walking

\section{Wearable devices and ICT systems}

Among the research activities, we have also developed a novel textile-based monitoring system (MagIC) [17], for acquisition and storage of physiological signals (ECG, respiration, and motion) recorded in patients at home or during outdoor activities, and for their remote transmission.

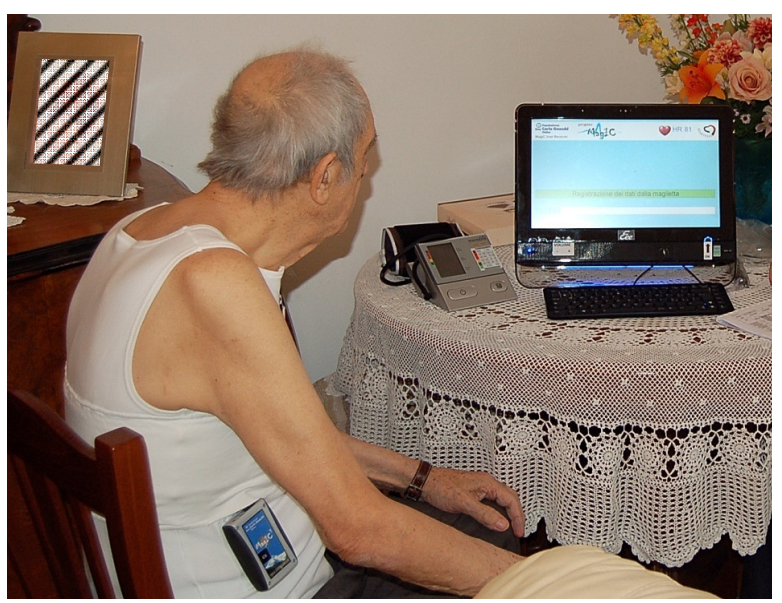

Figure 2. Example of the home monitoring system (from [18])

The system has been designed to be unobtrusive and easy to use, and it allowed us to explore the influence of the autonomic neural system on cardiorespiratory variables. Since 2004, MagIC has been used in many different clinical and non clinical conditions [19]. Moreover, a prototype version of this system has also been employed for the evaluation of motor performances in elderly patients suffering from Alzheimer disease [20]. Recently, the system has been companioned with a touch-screen PC running a simple multimodal software (allowing a mix of graphical, voice messages, touch-based interactions), for the home monitoring of heart failure patients (Fig.2). This architecture also allows clinicians to remotely administer tests on the patients' general health status and quality of life. This system 
was also proven to represent an effective telemedicine solution [18].

\section{Potential Role of ICT Pervasive Solutions}

The exponentially growing capabilities of data processing and communication of advanced portable and wearable devices are leading to radically new scenarios in our daily life. However, at present, little is known about possible usefulness of these solutions with respect to the NDDs management and follow-up: although some surveys are available, they are mainly focused on the AT solutions in specific NDDs [21-23] .

There are two trends of evolving ICT solutions which can give important contributions in the field. On the one side, by allowing a continuous monitoring of patient's and caregiver's conditions; on the other side, by establishing new communication channels between them and the clinical structures, or also between them and the rest of the society, even by means of the so-called social networks. The first trend regards miniaturization, reduction in costs and technical improvements of sensors, processing units, communication devices and interfaces. This is enabling unobtrusive continuous acquisition of information, from patient's physiological measures (ECG, respiratory signals, GSR, pulseoximetry, etc.) or motion-related quantities (accelerometers, GPS, magnetometers, gyroscopes, etc.). This information might be used to provide local real-time feedback, and can be also conveyed to a suitable repository for further processing. Such process allows clinicians to achieve a continuous insight of patient's conditions, or simply to be alerted in case of important changes in daily measures. This may lead to a more reactive care to patients, for example through a better and faster fine tuning of the pharmacological treatments, and, hopefully, to an overall enhancement of patients' quality of life. The second trend regards the evolution and more pervasive nature of communication devices. This may supports new approaches for assisting patients along the degeneration process of the disease. Synchronous communications, just like voice call or videoconferences with clinicians and caregivers, and also asynchronous ones, like emails, text messages etc. might indeed play a key role in "keeping in touch", helping patients to cope with their ever-changing disease, and continuously supporting their caregiver in everyday hurdles. In particular, if asynchronous communications are used, therapists, clinicians or remote operators are not required to be always online, waiting for their patients to connect, but rather they may receive alerts or daily/weekly reports. This in turn may help to lower the costs of such services.

Our recent experience with heart failure patients [18] highlighted that even establishing this kind of "low-cost" communication enhances the perception of being "supervised" or "in the network". This may prevent the feeling of being left alone, an issue often associated to NDDs. Moreover, the increasing capabilities and diffusion of mobile solutions (e.g. smartphones and tablet PCs) allows the same level of communication even outside the usual home boundaries. Looking ahead, interesting perspectives could be also related to interactions with social networks (like Facebook, Twitter, etc.). This can be achieved by means of usual computer or smartphone interfaces (when applicable), or through custom simplified multimodal solutions, like touchscreen adapted for users with reduced abilities. The value of this option is that it sustains the perspectives of (social) inclusion, by using widespread means, which already connects million of people.

\section{THE PROPOSED ARCHITECTURE}

A possible implementation of the ideas previously introduced, can be seen in Fig. 3. The architecture is composed by several communication endpoints (the interfaces of users with the system), that are linked via Internet connections, and a server collecting, processing and dispatching data. The endpoints may consist of normal PCs, PDAs, tablets or even smartphones, running suitable software applications. This software would allow to transfer two kind of information to the system: low level quantitative and high level .

Low level quantitative data would be provided by small and portable acquisition devices around or worn by the patients, wireless connected to the endpoint, or even by the endpoint itself, when containing sensors (i.e. recent smartphones equipped with accelerometers etc.). These devices would acquire and transfer quantitative data from a large number of different signals, physiological (i.e. ECG, respiration, EMG, etc.) kinematic (accelerometer, gyroscopes, magnetometer, GPS based, etc.) or environmental (light, temperature, pressure, humidity, etc.). This information, as well as other interesting measures derived from it (i.e. daily energy expenditure, postural states, etc.) would then be transferred automatically to the server, according to suitable secure policies, and coping with all the needed privacy issues. This may provide the system with enough data to trigger alert in case of subclinical worsening of health conditions, allowing early interventions and more personalized fine-tuning of the pharmacological and physical therapies.

Communication endpoints would be also used to distribute high level information, just like text, audio and video communication or data regarding the cognitive and psychological conditions of patients and caregivers. They would also allow the administration of surveys and verbal assessments to patients and caregivers.

The user interaction with the system through the communication endpoint would be based on simple multimodal and friendly applications at home or elsewhere, via smartphones. These communications may be synchronous (i.e. phone calls, chat or videoconferences, etc.) or asynchronous (SMS, mail, messages, etc.), thus allowing a good degree of flexibility and freedom. Hopefully, it would also help to reduce costs, because it doesn't require a $24 \times 7$ availability of operators or clinicians.

Patients and caregivers may take advantage of distributed pervasive solutions by being connected not only to clinicians or therapists, but also to other people, included NDDs groups. This would be possible not only at home, as previously described.

Such architecture may be help to manage and fine-tune usual AT systems used by the patients, by directly connecting them via suitable digital or analog interfaces 
when available, or indirectly, by means of the help of caregivers.

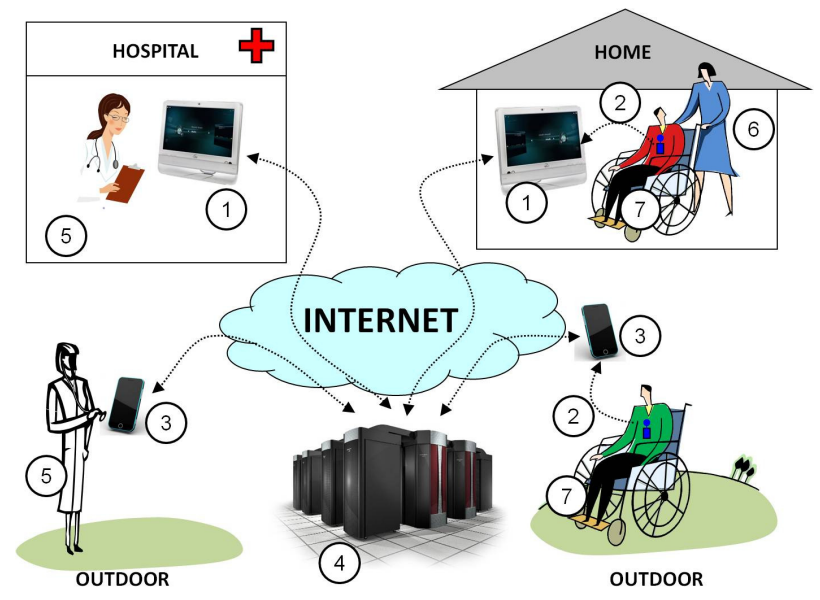

Figure 3. A comprehensive scheme of possible architecture: (1) communication endpoint (2) wearable acquisition devices which send data to the system. (3) mobile communication endpoint, (4) repository server,

(5) clinicians/therapists/remote operators (6) caregiver (7) patients

Of course, the success of the architecture we are proposing heavily depends on how the users would perceive it, and if and how often they would be ready and comfortable in using it. For this reason, it would be of great importance to perform careful usability and ergonomics analysis at a very early stage of the design process. Finally, another relevant issue would be the training phase of all the different users (patients, caregivers, operators, clinicians).

\section{CONCLUSIONS AND FUTURE WORK}

Although some technological solutions are already available, there is still an unmet demand of more tailored, responsive and flexible models of care for people with NDDs and of support for their caregivers [2]. On the base of the experience in the Assistive Technology, Neuromotor and Wearable Technologies of our department, we are addressing these issues by working on wearable technology and on simple multimodal communication endpoints with audiovideo communication channels and asynchronous exchange of information. We believe that these technological solutions might significantly help the follow-up of NDD-patients.

Indeed such kind of architecture lets clinicians to receive frequent or even continuous insights of the patient's conditions, and lets patients and caregivers to have simple and valuable communication channels with clinicians. Moreover, a proficient use of these communication means might empower the social inclusion of patients and caregivers. This will not be limited to home or hospital boundaries, but will be open also to outdoor. In this perspective, a relevant role is related to the real usability of the architecture by the users, as well as careful analysis of its efficiency and efficacy. This approach may open interesting perspectives in the health management of NDD patients, and may provide significant improvement in patients' and caregivers' quality of life. Of course, legal, economic, organizational and privacy issues are aspects to be further addressed to translate this methodology in clinical practice.

\section{REFERENCES}

[1] F. Patti, et al., "Caregiver quality of life in multiple sclerosis: a multicentre Italian study," Mult Scler, vol. 13, pp. 412-9, Apr 2007.

[2] S. Aoun, et al., "Caregivers of people with neurodegenerative diseases: profile and unmet needs from a population-based survey in South Australia," J Palliat Med, vol. 13, pp. 653-61, Jun 2010.

[3] K. Lode, et al., "Coping with multiple sclerosis: a 5-year follow-up study," Acta Neurol Scand, Dec 282009.

[4] "Technology-related Assistance for Individuals with Disabilities Act,", 1988, P.L. 100-407

[5] A. A. Conti, "Modern rehabilitation in Italy: the lesson of Father Carlo Gnocchi," Am J Phys Med Rehabil, vol. 87, pp. 687-9, Aug 2008.

[6] C. L. Cazzullo, "History and activities of the Don Carlo Gnocchi multiple sclerosis center," J Neurovirol, vol. 6 Suppl 2, p. S4, May 2000.

[7] R. Andrich and A. Caracciolo, "Analysing the cost of individual assistive technology programmes," Disabil Rehabil Assist Technol, vol. 2, pp. 207-34, Jul 2007.

[8] R. Andrich, et al., "The DAT project: A smart home environment for people with disabilities," Computers Helping People with Special Needs, Proceedings, vol. 4061, pp. 492-499, 2006.

[9] M. Ferrarin, et al., "Effects of bilateral subthalamic stimulation on gait kinematics and kinetics in Parkinson's disease," Exp Brain Res, vol. 160, pp. 517-27, Jan 2005.

[10] I. Carpinella, et al., "Locomotor function in the early stage of Parkinson's disease," IEEE Trans Neural Syst Rehabil Eng, vol. 15, pp. 543-51, Dec 2007.

[11] P. Crenna, et al., "Influence of basal ganglia on upper limb locomotor synergies. Evidence from deep brain stimulation and L-DOPA treatment in Parkinson's disease," Brain, vol. 131, pp. 3410-20, Dec 2008.

[12] M. Ferrarin, et al., "Procedure for the quantitative evaluation of motor disturbances in cerebellar ataxic patients," Med Biol Eng Comput, vol. 43, pp. 349-56, May 2005.

[13] I. Carpinella, et al., "Robot-based rehabilitation of the upper limbs in multiple sclerosis: feasibility and preliminary results," J Rehabil Med, vol. 41, pp. 966-70, Nov 2009.

[14] R. Pignatti, et al., "Specific impairments of selective attention in mild Alzheimer's disease," J Clin Exp Neuropsychol, vol. 27, pp. 436-48, May 2005.

[15] M. Ferrarin, et al., "Reliability of instrumented movement analysis as outcome measure in Charcot-Marie-Tooth disease: results from a multitask locomotor protocol," Gait Posture, p. in press, 2011.

[16] M. Rabuffetti, et al., "Touch-screen system for assessing visuomotor exploratory skills in neuropsychological disorders of spatial cognition," Med Biol Eng Comput, vol. 40, pp. 675-86, Nov 2002.

[17] M. Di Rienzo, et al., "MagIC System: a New Textile-Based Wearable Device for Biological Signal Monitoring. Applicability in Daily Life and Clinical Setting," Conf Proc IEEE Eng Med Biol Soc, vol. 7, pp. 7167-9, 2005.

[18] P. Meriggi, et al., "A new simple multimodal platform for home monitoring of cardiac patients through textile technology," in Computers in Cardiology, Park City, Utah, USA, 2009.

[19] M. Di Rienzo, et al., "Textile technology for the vital signs monitoring in telemedicine and extreme environments," IEEE Trans Inf Technol Biomed, vol. 14, pp. 711-7, May 2010.

[20] P. Castiglioni, et al., "A New Wearable System for Movement Analysis: Application in Elderly Subjects with and without Alzheimer Disease," in World congress on Medical Physics and Biomedical Engineering 2006, Seoul, Korea, 2006.

[21] D. J. Blake and C. Bodine, "An overview of assistive technology for persons with multiple sclerosis," J Rehabil Res Dev, vol. 39, pp. 299-312, Mar-Apr 2002.

[22] K. L. Johnson, et al., "Use of cognitive aids and other assistive technology by individuals with multiple sclerosis," Disabil Rehabil Assist Technol, vol. 4, pp. 1-8, Jan 2009.

[23] A. Souza, et al., "Multiple sclerosis and mobility-related assistive technology: systematic review of literature," J Rehabil Res Dev, vol. 47, pp. 213-23, 2010. 